\title{
Voice Operated Switch Design of Smart Home Based on Single Chip Microcomputer Technology
}

\author{
Lei Yao \\ Chengdu Polytechnic, Chengdu, Sichuan, 610041, China \\ 565169780@qq.com
}

Keywords: Speech chip; Single chip microcomputer(SCM); Relay; Smart home

\begin{abstract}
The purpose of this paper is to design a voice operated switch which is widely used in smart home. The core control part of the voice intelligent switch is the single chip microcomputer(SCM). By processing the information collected by the voice chip, it controls the lighting of the home and the opening and closing of the curtains. Through this design, the organic integration of the subsystems related to home life is finally achieved, which ultimately makes our lives more intelligent, comfortable and humane.
\end{abstract}

\section{Introduction}

The rapid development of new intelligent products cannot be separated from the continuous development of science and technology and the popularization of single chip microcomputer knowledge. The platform of the smart home is a house. The main technologies used are integrated wiring technology, automatic control technology, and computer technology. It integrates various subsystems related to home life through various devices and methods, and ultimately realizes the intelligence, safety and comfort of the home environment.

Voice control refers to the control of the device through the human voice. When the device receives the human voice, it can convert the words in the voice into an input sentence that can be recognized by the computer, thereby receiving instructions and implementing the functions of the voice control device.

Modern people's work pressure and life pressure are relatively large, and the intelligence of life demands higher requirements. I designed this smart home voice operated switch in order to bring convenience to people's lives.

\section{The Status of Smart Home at Home and Abroad}

The basic purpose of smart home is to make the living environment more comfortable, more convenient, safer and more intelligent. With the development of economy and the continuous improvement of people's consumption level, the future smart home will have richer contents and system design will be more and more complicated. In some developed countries, smart home has developed greatly. They laid the foundation for the development of intelligent furniture, and also standardized the technical standards. However, due to the difference of the national economic level and ideology, the requirements for smart home are different. Smart homes in the United States take digital home and digital technology as an opportunity to focus on comfort and enjoyment, but the results consume huge energy sources and violate the concept of energy conservation, low carbon and environmental protection. In Japan, they pay more attention to users' feelings, adopt new technology, make full use of information, network, intelligent control and artificial intelligence technology, and take into account the function of environmental protection. The German smart home is pursuing the development of the patent function, paying attention to the basic function. South Korea encourages and supports the development and construction of smart communities and smart homes. At present, more than $80 \%$ of new projects using smart home systems, such as the well-known smart home brands Samsung, LG and so on.

At present, the domestic manufacturers of smart home can be divided into two categories: one is the factory designed to live and work, their main design areas are automatic alarm, environmental 
monitoring, security monitoring and so on. The other is the manufacturer of household appliances. These manufacturers mainly standardize the household appliances and standardize the household appliances. On the basis of the platform, the intelligent home appliances network is built. In addition, there is a class of production focused on peripheral equipment and devices. They are mainly combined with the first two types of manufacturers and provide various kinds of intelligent switches and interface modules. To some extent, the development of domestic smart home industry is restricted because the domestic related industry technical standards have been unable to be unified, although there are many smart home control system products.

There are many factors that restrict the development of the domestic smart home market. The main ones are the inconsistency of standards, the complexity of the system, the lack of aesthetics, the imperfect after-sales service, and the high prices.

\section{Speech Recognition Technology}

Although the specific implementation details of different speech recognition systems are different, the basic technologies used are similar. A typical implementation of the speech recognition system is shown in Fig. 1.

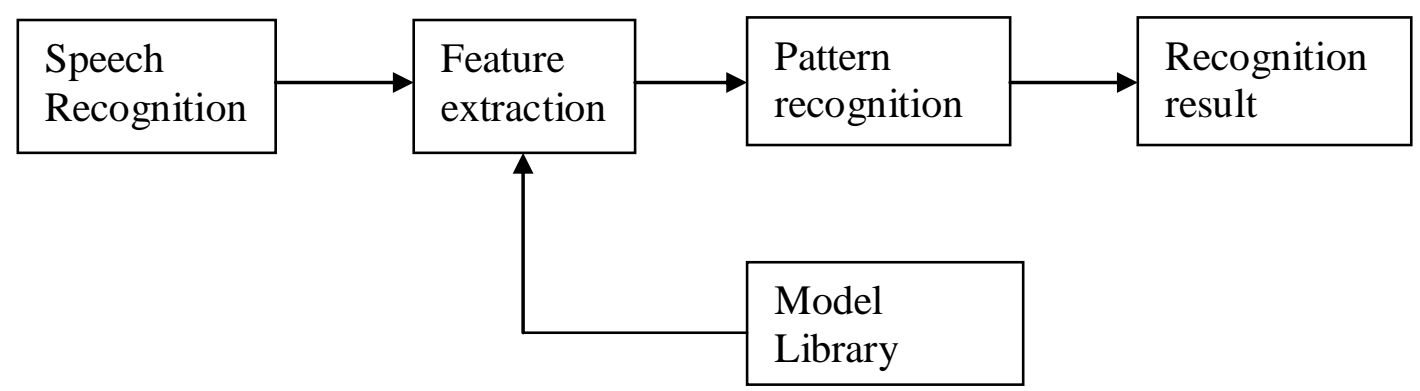

Figure 1. Finite The Speech Recognition System Implementation Diagram

Speech Recognition ASR technology is to set up a list of key words to be recognized and transfer these key words in the form of characters to the inside of the LD3320. The user does not need to do any recording training. Therefore, the main technology of speech recognition is the keyword list recognition technology.

\section{The Overall Program Design}

The smart home voice operated switch designed here mainly uses advanced non-specific human voice recognition technology, analyzes the identified content, and issues control instructions to control the controllable devices in the smart home so as to achieve more comfort and personality.

System Block Diagram. The whole system mainly includes two parts: core main control and speech recognition. The hardware circuit is divided into four parts, voice recognition module, STC89C52 microcontroller minimum system, 4-way relay module and LED lights. The core main control part is the minimum system of the STC89C52RC microcontroller. The voice recognition part includes the STC11L08XE microcontroller and the LD3320 voice recognition chip. The STC11L08X microcontroller is connected to the LD3320 through parallel mode and is responsible for the control of the LD3320 and is externally connected to the output port of the minimum system control voice module of the STC89C52RC microcontroller in order to control the on and off of the LED lights and the switching of the relays, thereby further controlling the switch of curtains, air conditioners and other furniture. The system block diagram is shown in Fig. 2. 


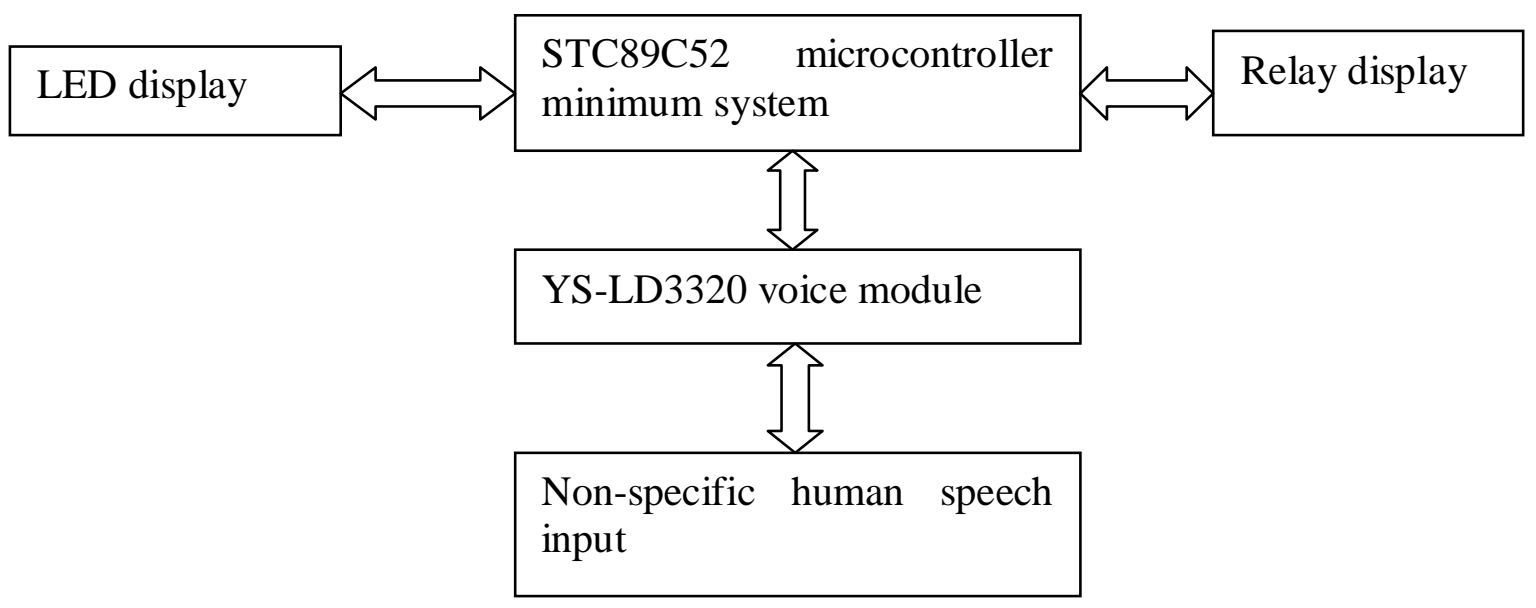

Figure 2. Finite The system block diagram

Circuit Principle. The VCC and GND of the voice recognition module, the minimum system of the STC89C52 microcontroller, and the 4-way relay module are respectively connected to the common power supply VCC and GND on the circuit board. The external USB interface is connected to the VCC and GND of the common power supply to ensure the power supply of the entire circuit.

In addition, the P2.3 and P2.1 ports of the minimum system of the STC89C52 microcontroller are connected to the P2.3 and P2.4 ports of the voice recognition module, respectively. The P2.2 port of the minimum system of the STC89C52 microcontroller is connected to the IN1 port of the 4-way relay module. P2.0 port is connected to the positive pole of the LED light, and the negative pole is connected to the negative pole of the resistance. The positive pole of the resistor is connected to the power supply VCC. The role of this resistor is to prevent excessive current from burning LED lamps.

Specifically, the P2.3 and P2.4 ports of the speech recognition module are defined. The P2.3 port controls the on and off of the LED lights. The P2.4 port controls the opening or closing of the relay. When the microphone receives the "turn on the light"/"turn off light"/"turn on relay"/ "turn off relay" commands, the commands are sent to the P2.3 and P2.4 ports of the voice module. Turn on the light at low level, let P2.3 low level; Turn off the light at high level, let P2.3 high level; Open the relay at low level, let $\mathrm{P} 2.4$ low, close the relay at high level, let P2.4 high. The command is input to the P2.3 and P2.1 ports of the minimum system of the STC89C52 microcontroller and the P2.3 and P2.4 ports of the SCM control voice module. Finally, it is output to the IN1 port of the four-way relay module through the P2.2 port of the minimum system of the STC89C52 microcontroller, and then to the P2.0 port, and finally to the LED lights. Relays and LED lights received commands to open or close. Circuit schematics and physical maps are shown in Fig. 3 and Fig. 4. 


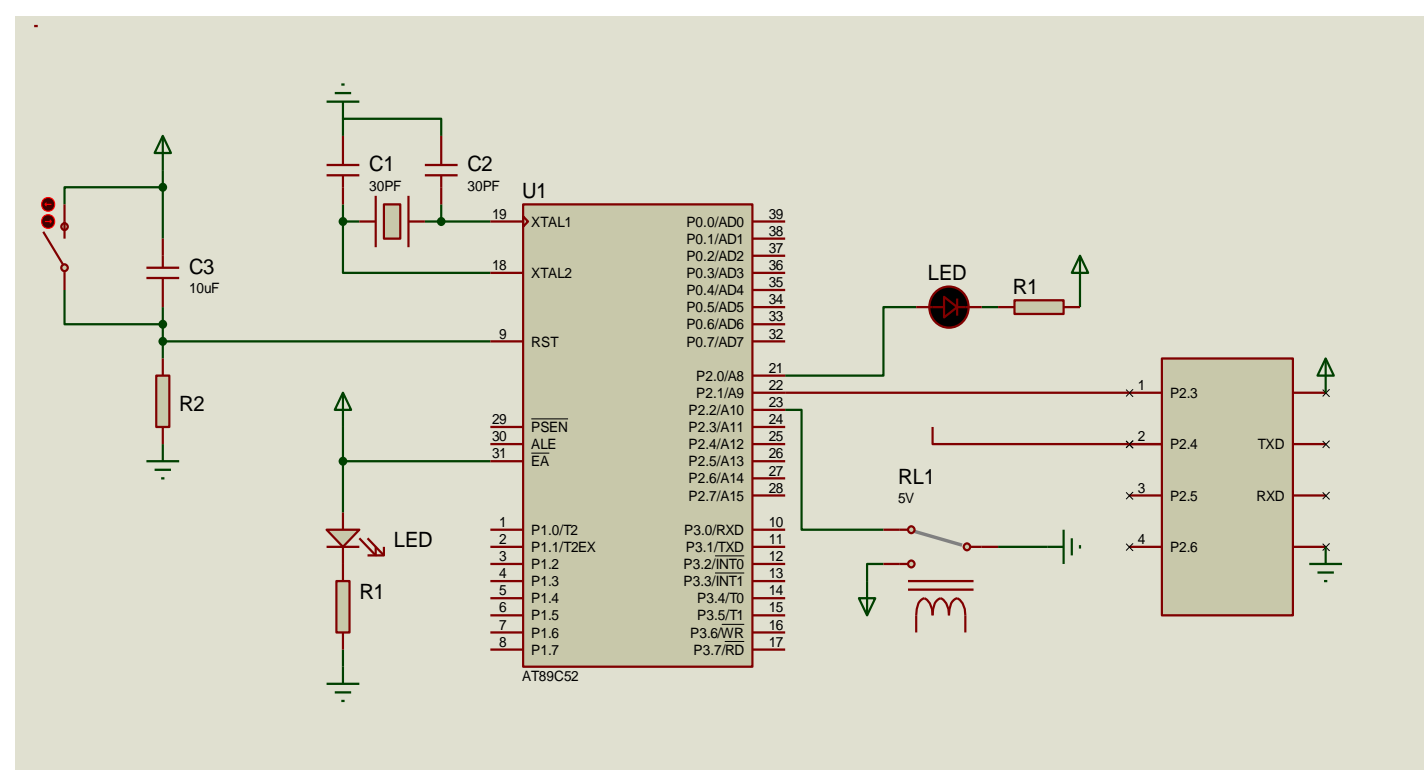

Figure 3 The Overall circuit schematic map

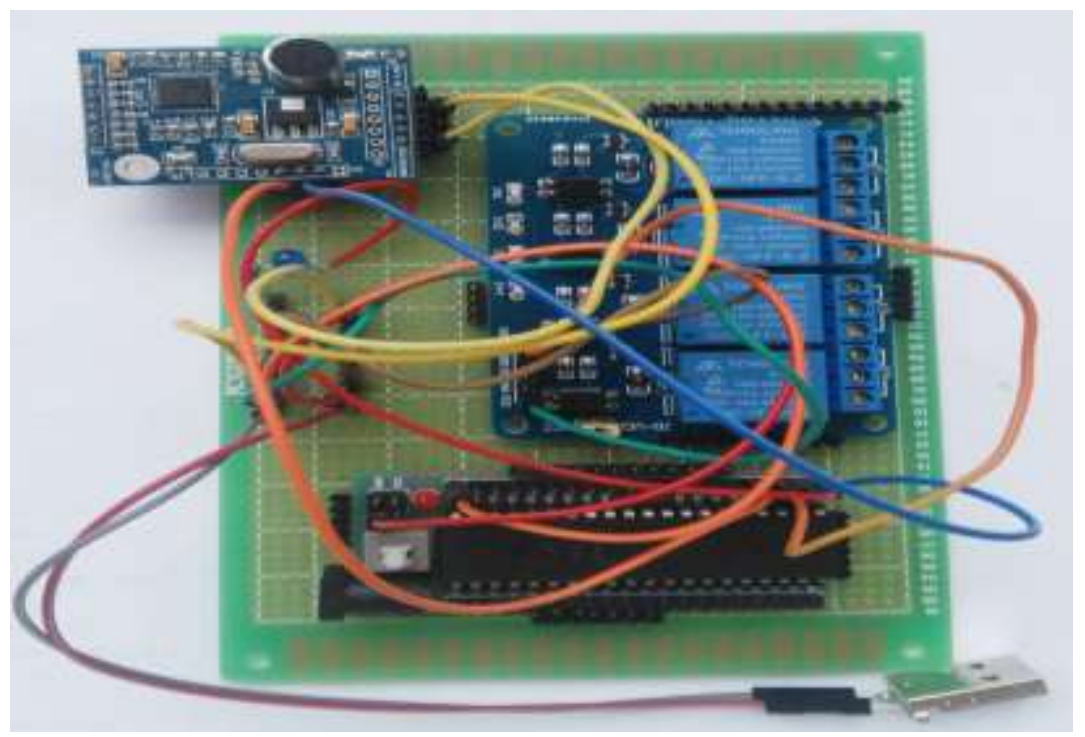

Figure 4. Finite The physical map

Software Design. This article uses Keil software to program code, requires the support of WIN98, NT, WIN2000, WINXP and other operating systems, its integrated environment is easy to use, and software simulation and debugging tools are also very powerful.

The program mainly includes the following parts: Application IO port definition, ASR identification, LED lamp test, SCM initialization, delay function, interrupt handling function, and user execution function.

Application IO port definition: It defines the port of the control relay and LED lamp on the module, and then initializes the serial port without ASR.

ASR recognition: It sets up a list of key words to be recognized and transmits these keywords in the form of characters to the inside of the LD3320.

LED lamp test: It is used to detect whether the microcontroller has working instructions, and delay $200 \mathrm{~ms}$.

User-executed function: It controls the high and low levels of the PA port to control the on and off of external relays and the on and off of LED lights. Program flow chart is shown in Fig. 5. 


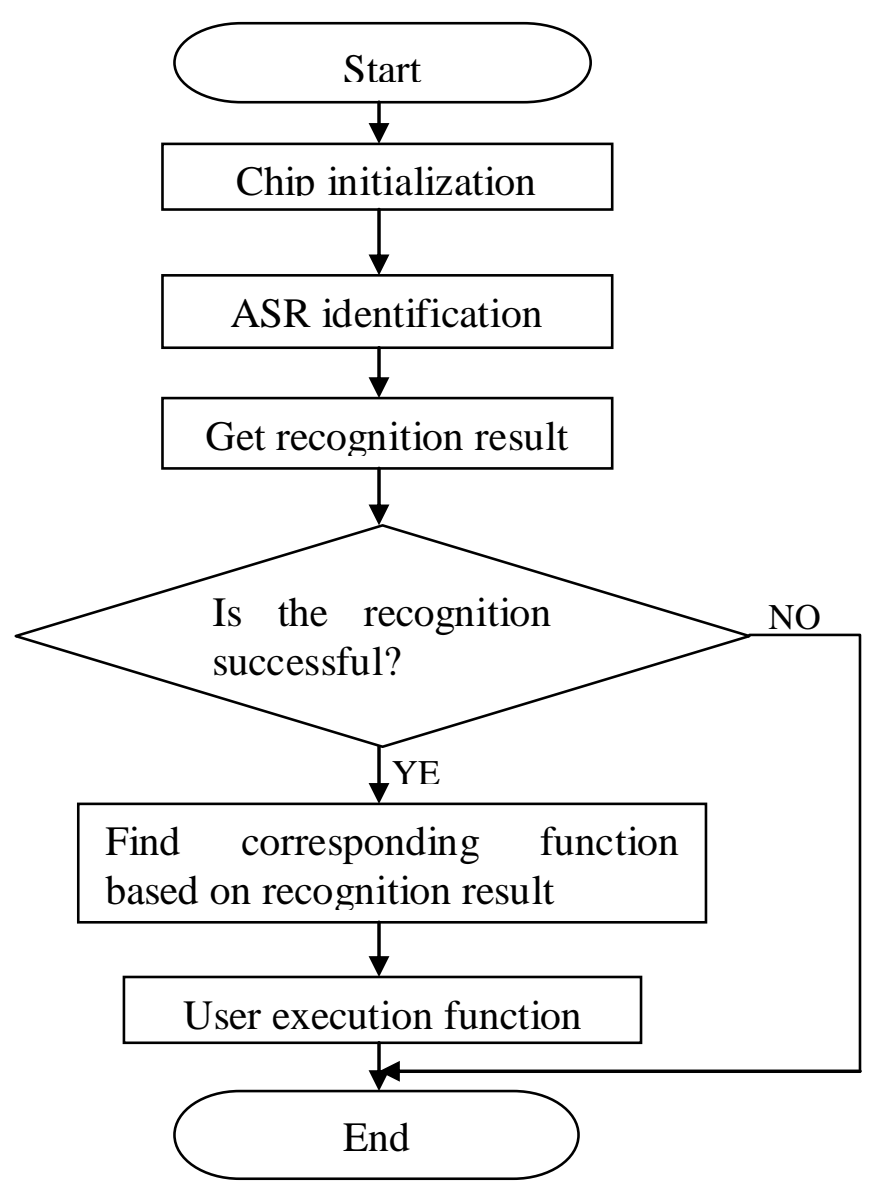

Figure 5. Finite The Program flow chart

After the program starts, the chip initialization is mainly responsible for setting the corresponding register. After initialization, the LD3320 voice chip performs ASR identification on the commands collected by the microphone. ASR adds keywords and the ASR operation is started. After the ASR identification process is completed, the ASR recognition result is obtained. If the speech recognition is successful, it will find the corresponding function according to the speech recognition result. Once the user execution function is found, the MCU will start working. It will drive the relay on or off, and control the LED lights on or off. If speech recognition is unsuccessful, the program will not continue to run and the MCU will not work. The end result is no response from the relay and LED lights.

\section{System Debugging}

Synthetic Debugging of Hardware and Software. Before the debugging, the hardware circuit should be designed well. After the program is written (omit the program code), the USB data line is used to download the designed target program to STM11L08XE microcontroller and STC89C52RC microcontroller on the target board for block debugging: serial port debugging, voice module debugging, and minimum system debugging of SCM. Through hardware, we can see whether the functions required by the subject can be got, and the two combine debugging. First, we should debug the circuit to read and write registers correctly, and communicate with the LD3320 chip. If the users fail to read and write registers correctly, they should check the hardware connections carefully, including welding, resistance, crystal oscillator and so on, according to the above schematic diagram.

In debugging, the general inspection sequence is: Measure and check whether the voltage supplied to the LD3320 chip is correct: the VDD, VDDIO, and VDDA three-channel power supply pins need to be connected to the $3.3 \mathrm{~V}$. The two-channel ground pins need to be properly grounded. 
According to the transmission mode (parallel port or SPI), we should measure whether the voltage of MD and SPIS pin is correct.

\section{Debug Steps.}

The first step is to detect the minimum system of SCM.

The second step is whether the voice chip can recognize the voice.

The third step is whether the LED lamp works normally controlled by the SCM.

The fourth step is whether the relay works normally controlled by the SCM.

The fifth step is to display a variety of data processing.

The sixth step is the overall circuit connection, the overall debugging, and to solve the detail problems.

The seventh step is to test the performance of the system.

Debug Results. When the USB interface is connected to the computer, in the voice module, one indicator light flashes three times, and one indicator light is always on, and the indicator light on the minimum system of SCM is on, indicating that the module and the SCM work normally, as shown in Fig. 6.

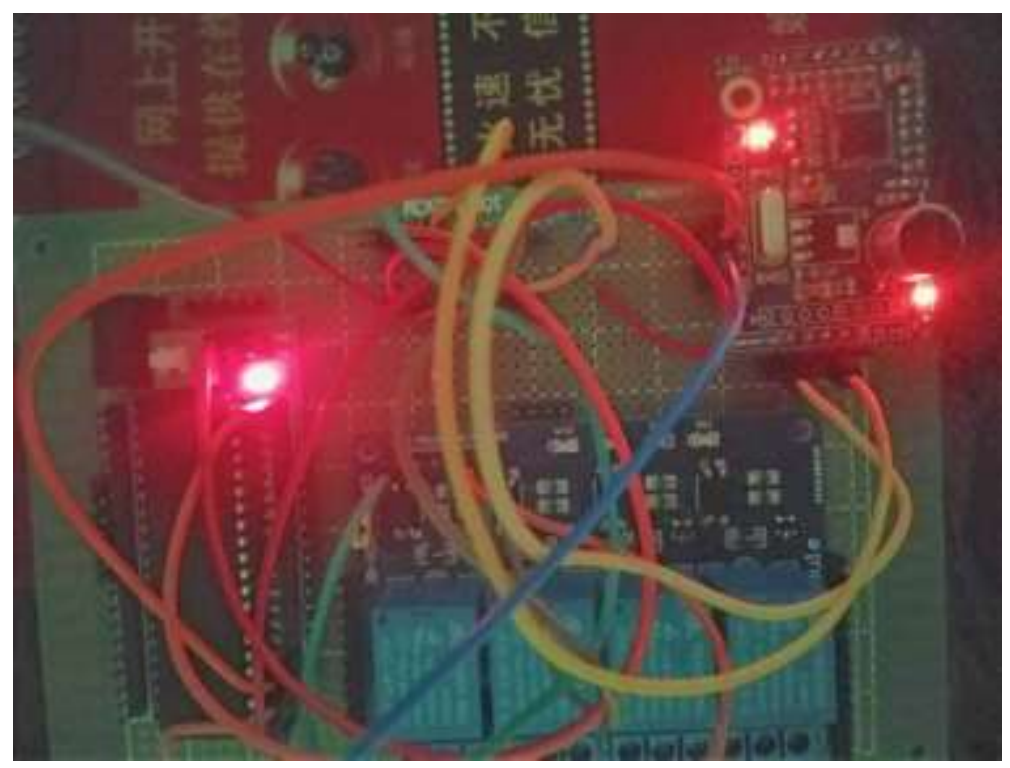

Figure 6. Finite The physical map of power on

When the "turn on the light" / "turn off the light" command is said, the indicator light is always on in the voice module, the speech recognition is successful, and the LED lights are on/off, as shown in Fig. 7 and Fig. 8. 


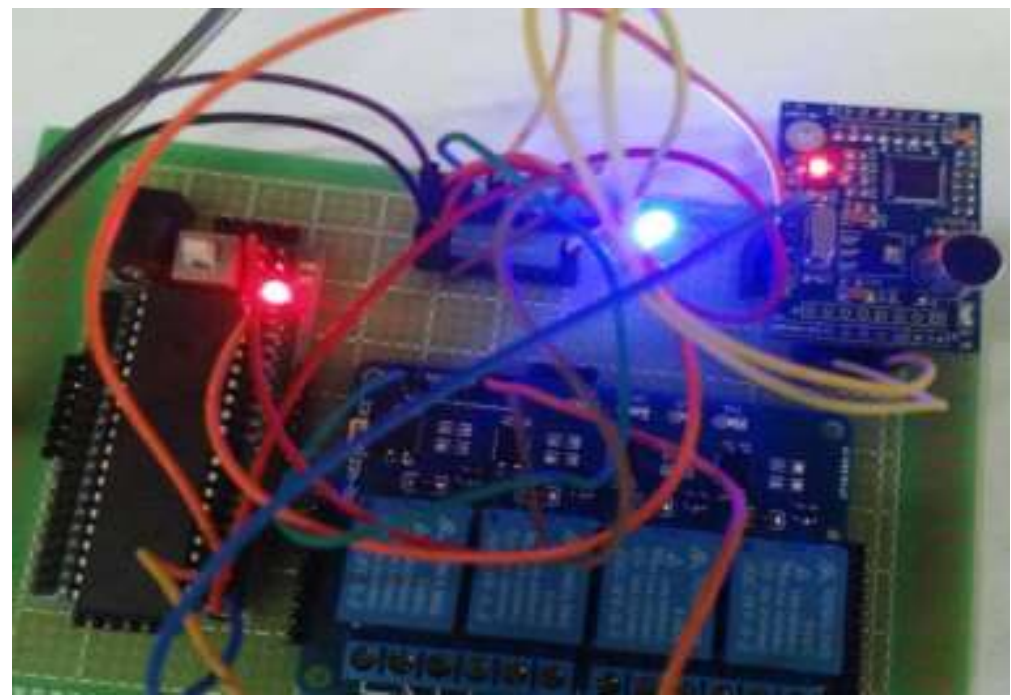

Figure 7. Finite Turn on the light figure of speech recognition

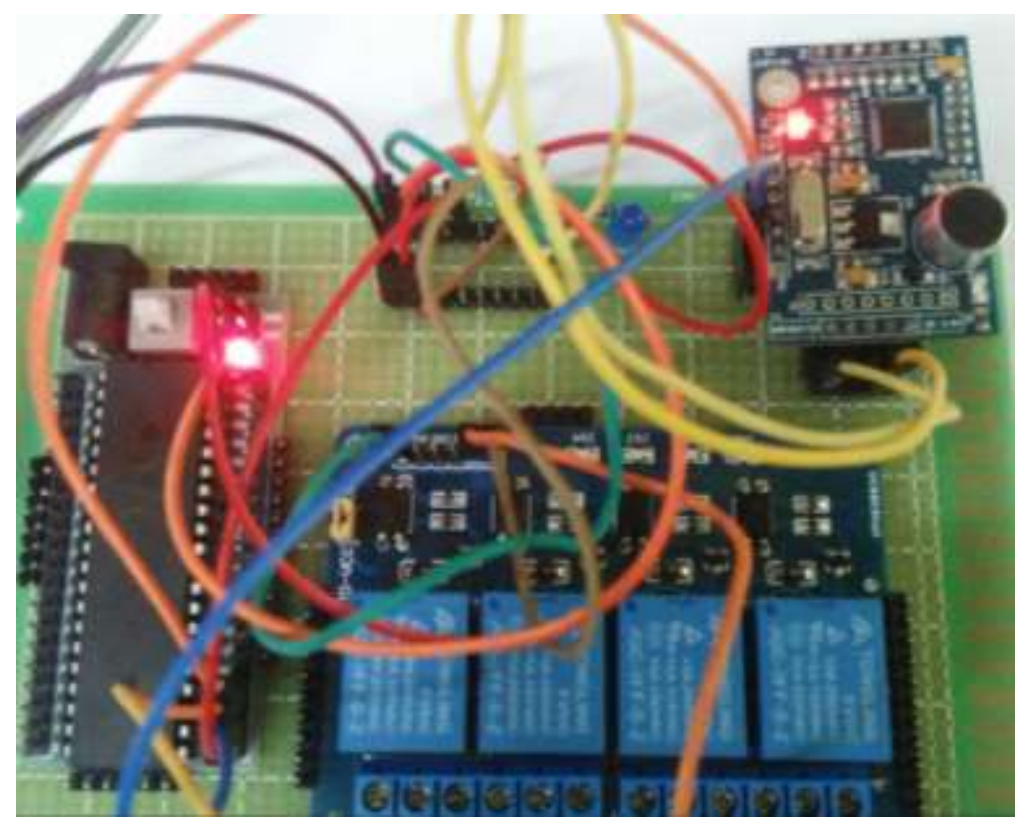

Figure 8. Finite Turn off the light figure of speech recognition

When saying "open the relay" / "turn off the relay" command, the indicator light on the voice module is always on, the speech recognition is successful, at the same time, the relay indicator light is on / off and makes a clear sound, the figure is omitted.

In different environments, the test results will also be biased. When testing in a quiet environment, the recognition effect is better. When the first command is made, the LED lamp and relay will react. However, when testing in a noisy environment, the recognition results are poor. When the first command is made, it may not be recognized, and the LED lights and relays do not respond. In this case, it can be identified again after several tries, and LED lights and relays will react.

The sound level also affects the test results. If the voice is larger, the recognition effect will be better. Once a command is spoken, they will instantly recognize and react. Conversely, if the voice is small, the recognition effect is poor, and the command needs to be repeated several times before they can recognize and respond. 


\section{The Advantages and Disadvantages of Design and Its Prospect}

Although the system has introduced the speech recognition module, the single chip microcomputer and so on, for the real meaning of the comprehensive intelligent home life, it is still very inadequate. According to the results of the above test, we hope to improve the follow-up design.

The first one is the diversity of language recognition. We can design a voice operated switch which can not only recognize mandarian, but also recognize dialects across the country. This kind of voice operated control switch will be more widely used.

The second one is the sustomization of speech recognition. Voice operated switches can be controlled not only by specific words, but also by clapping, hitting fingers and so on.

The third one is the anti-interference and sensitivity of speech recognition. The intelligent home voice operated switch designed in this paper is still in the experimental stage, and there is still a big gap from the practical application. After that, we will further improve the circuit and program design, improve the reliability of the communication of the intelligent home system and reduce the energy consumption of each module, and strive to contribute to the creation of intelligent humanized home life.

\section{Acknowledgement}

Scientific Research Project Funded by the Sichuan Provincial Education Department "Research on Data Security System Based on Power Architecture"(18ZA0171)

\section{References}

[1] Ying $\mathrm{Xu}$, Chunzhi Sun and Chenguang Wu: Design of Integrated Voice Control System for Smart Home Based on LD3320[J], Journal of Shangqiu Normal University, 2016-06-15.

[2] Peng Su, Fengyu Zhou and Lei Chen: Design of Embedded Voice Recognition Module Based on STM32[J], Microcontroller \& Embedded Systems, 2011-02-01.

[3] Wei Fu, Pengguang Tang and Qian Li: Design of Intelligent Home Voice Control System[J], Process Automation Instrumentation, 2014-01-20.

[4] Digital Voltmeter Course Design of Single Chip Microcomputer, "Internet Document Resources (http://wenku.baidu.c)", 2016-11-14.

[5] Design of Timing Socket Based on Single Chip Microcomputer, "Internet Document Resources (http://wenku.baidu.c)". 2017-3-21

[6] Graduation Thesis for Traffic Lights, "Internet Document Resources(http://wenku.baidu.c)", 2017-5-5.

[7] Tu Liu, Nengcao Feng and Rushi Zhu: Short Message Water Level Query System Based on SDI-12 Interface Water Level Gauge[J], Journal of Engineering of Heilongjiang, 2010-03-25.

[8] Gao Lv: Design of Wireless Communication Based on STC12LE2052AD and nRF2401 Module[J], Journal of Electric Power, 2007-12-15.

[9] Yunping Jiao: Multifunctional Ozone Shoe-Drying Machine Based on Single Chip Microcomputer[J], Science \& Technology Information, 2010-12-23.

[10] Zehao Tang, Zhibo Zhang and Jindong Wang: Research on Optocoupler Control Based on STC Single Chip[J], Digital Technology and Application, 2011-12-15.

[11] Jiaping Hong: Application of LD3320 Embedded Speech Recognition System[J], Application of Single Chip Computer and Embedded System, 2012-02-01.

[12] Yanhuan Cao: Smart Home Controller[J], Practical Electronics, 2015-12-01.

[13] Yuan Tian: Research and Design of Voice Control Playback System[J], Journal of Baotou Vocational \& Technical College, 2013-09-15.

[14] Xichun Chen: Speech Control Based on LD3320 Speech Recognition Special Chip[J], Electronic Technology, 2011-11-25.

[15] New Technology and New Process: Design and Implementation of Intelligent Blind Navigator[J], New Technology \& New Process, 2015-03-25. 